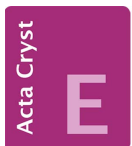

CRYSTALLOGRAPHIC COMMUNICATIONS

ISSN 2056-9890

Reçu le 4 avril 2015

Accepté le 5 mai 2015

Édité par W. T. A. Harrison, Université de Aberdeen, Ecosse

Keywords: crystal structure; lyonsite-type; monovalent cation molybdate; bond-valence calculations

CCDC reference: 1063336

Supporting information: this article has

supporting information at journals.iucr.org/e

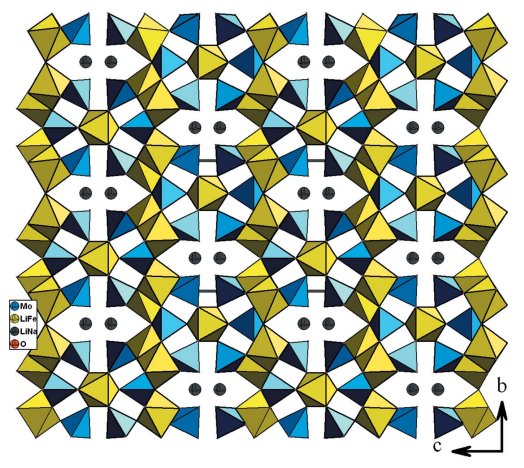

OPEN $\odot$ ACCESS

\section{Synthèse et étude structrale de lyonsite-type $\left(\mathrm{Na}_{0,4}, \mathrm{Li}_{0,6}\right)\left(\mathrm{Fe}, \mathrm{Li}_{2}\right)\left(\mathrm{MoO}_{4}\right)_{3}$}

\author{
Amira Souilem,* Mohamed Faouzi Zid et Ahmed Driss
}

Laboratoire de Matériaux et Cristallochimie, Faculté des Sciences de Tunis, Université de Tunis ElManar, 2092 Manar II Tunis, Tunisie. *Correspondence e-mail: souilem_amira@yahoo.fr

The new compound $\left(\mathrm{Na}_{0.4}, \mathrm{Li}_{0.6}\right)\left(\mathrm{Fe}, \mathrm{Li}_{2}\right)\left(\mathrm{MoO}_{4}\right)_{3}$ was synthesized by cooling from the melt. Its anionic framework is built up from two distinct $\mathrm{MO}_{6}$ octahedra, each containing disordered $\mathrm{Li}^{+}$and $\mathrm{Fe}^{3+}$ ions in 0.6:0.4 and 0.7:0.3 ratios, and two $\mathrm{MoO}_{4}$ tetrahedra, which link by vertex-sharing of their $\mathrm{O}$ atoms. These tetrameric units are further linked by sharing edges between octahedra and by formation of $M-\mathrm{O}-\mathrm{Mo}(M=\mathrm{Fe} / \mathrm{Li})$ bridges, forming ribbons propagating in the [100] direction. The ribbons are cross-linked in both the $b$ and $c$-axis directions, giving rise to a three-dimensional framework having [100] tunnels in which the monovalent $\mathrm{Na}^{+} / \mathrm{Li}^{+}$cations (0.4:0.6 ratio) lie. Bond-valence calculations are consistent with the disorder model for the cations. The structure of the title compound, which is isotypic with $\mathrm{Li}_{3} \mathrm{Fe}\left(\mathrm{MoO}_{4}\right)_{3}$ and $\mathrm{Li}_{3} \mathrm{Ga}\left(\mathrm{MoO}_{4}\right)_{3}$, is compared briefly with those of $\mathrm{LiFeMo}_{2} \mathrm{O}_{8}$ and $\mathrm{Li}_{1.6} \mathrm{Mn}_{2.2}\left(\mathrm{MoO}_{4}\right)_{3}$.

\section{Contexte chimique}

La famille des molybdates de cations monovalents est l'objet d'un grand intérêt ces dernières années à cause de l'importance de leurs propriétés physiques et les applications potentielles, prenant l'exemple des matériaux laser prometteurs, des luminophores efficaces, qui sont caractérisés par une longue durée de vie et une haute intensité de luminescence, ainsi que des matériaux ferroélectriques et antiferro-

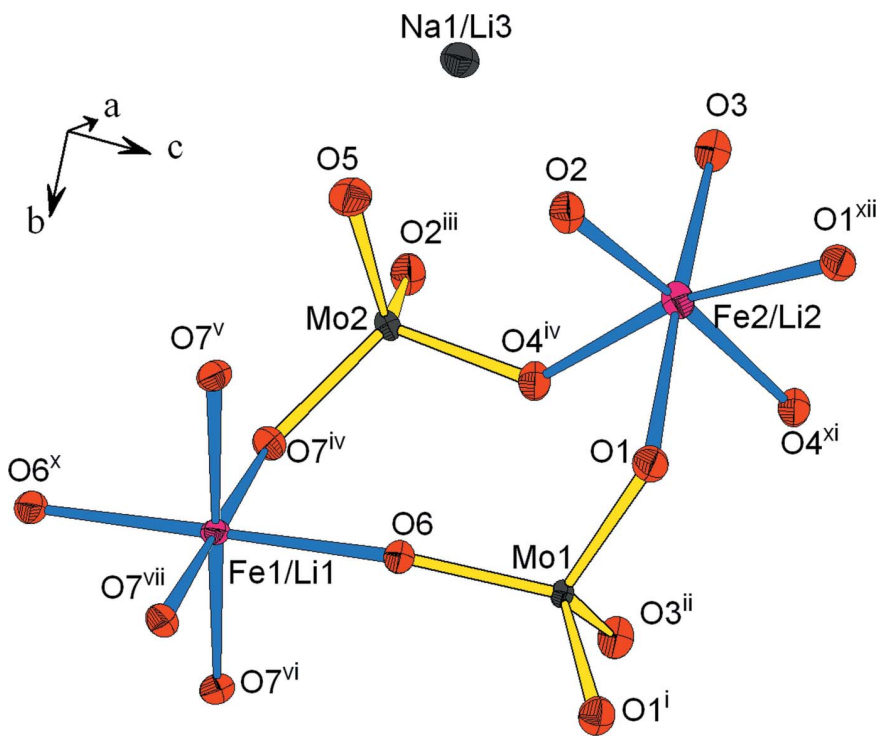

Figure 1

Représentation des polyèdres de coordination de l'unité structurale dans le composé $\left(\mathrm{Na}_{0,4}, \mathrm{Li}_{0,6}\right)\left(\mathrm{Fe}, \mathrm{Li}_{2}\right)\left(\mathrm{MoO}_{4}\right)_{3}$. [Codes de symétrie: (i) $x,-y+\frac{3}{2}$, $z$; (ii) $x, y, z+1$; (iii) $x+1, y, z$; (iv) $x, y+1, z$; (v) $x-\frac{1}{2},-y+\frac{1}{2},-z+\frac{1}{2}$; (vi) $x-\frac{1}{2}, y,-z+\frac{1}{2}$; (vii) $x-1, y, z$; (x) $-x+1,-y+1,-z+1$; (xi) $-x+1$, $-y+1,-z$; (xii) $x-1, y, z-1$.] 


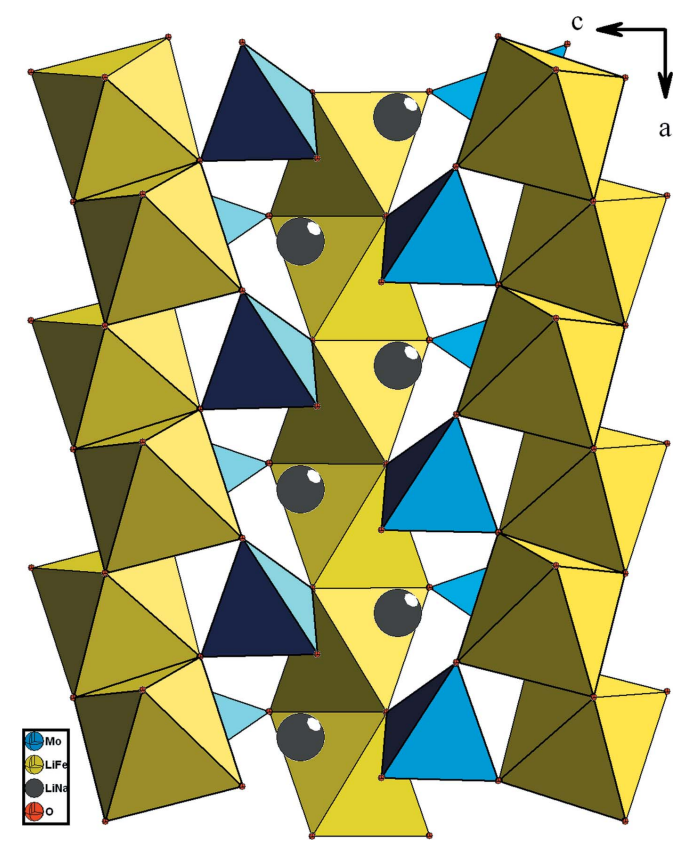

Figure 2

Forme d'un ruban selon $a$ dans le composé $\left(\mathrm{Na}_{0,4}, \mathrm{Li}_{0,6}\right)\left(\mathrm{Fe}, \mathrm{Li}_{2}\right)\left(\mathrm{MoO}_{4}\right)_{3}$.

magnétiques (Morozov et al., 2003; Isupov, 2005; Maczka et al., 2005; Jorge et al., 2004). L'étude des structures cristallines de ces molybdates souligne leur polymorphisme dépendant de la température (Klevtsov \& Klevtsova, 1977). De plus, les composés à base de polyanions sont actuellement proposés comme une alternative prometteuse aux matériaux de $\mathrm{Li} \mathrm{MO}_{2}$ $(M=\mathrm{Ni}, \mathrm{Co}, \mathrm{Mn})$ stratifiés comme cathode pour des piles rechargeables. L'utilisation du lithium est visé vu qu'il possède
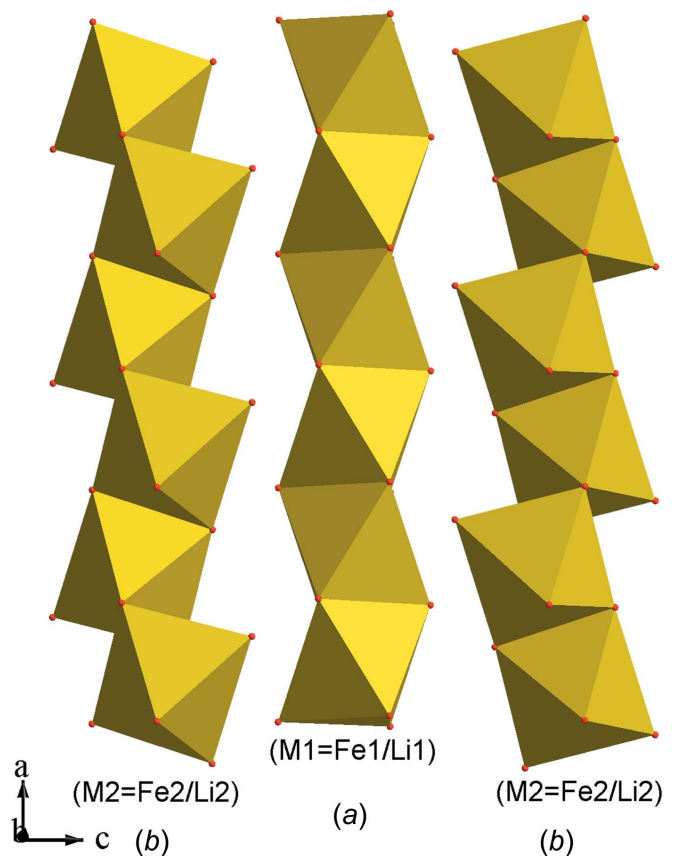

(b)

Figure 3

Chaînes d'octaèdres dans le composé $\left(\mathrm{Na}_{0,4}, \mathrm{Li}_{0,6}\right)\left(\mathrm{Fe}, \mathrm{Li}_{2}\right)\left(\mathrm{MoO}_{4}\right)_{3}$. (a): type $1 ;(b)$ : type 2 .

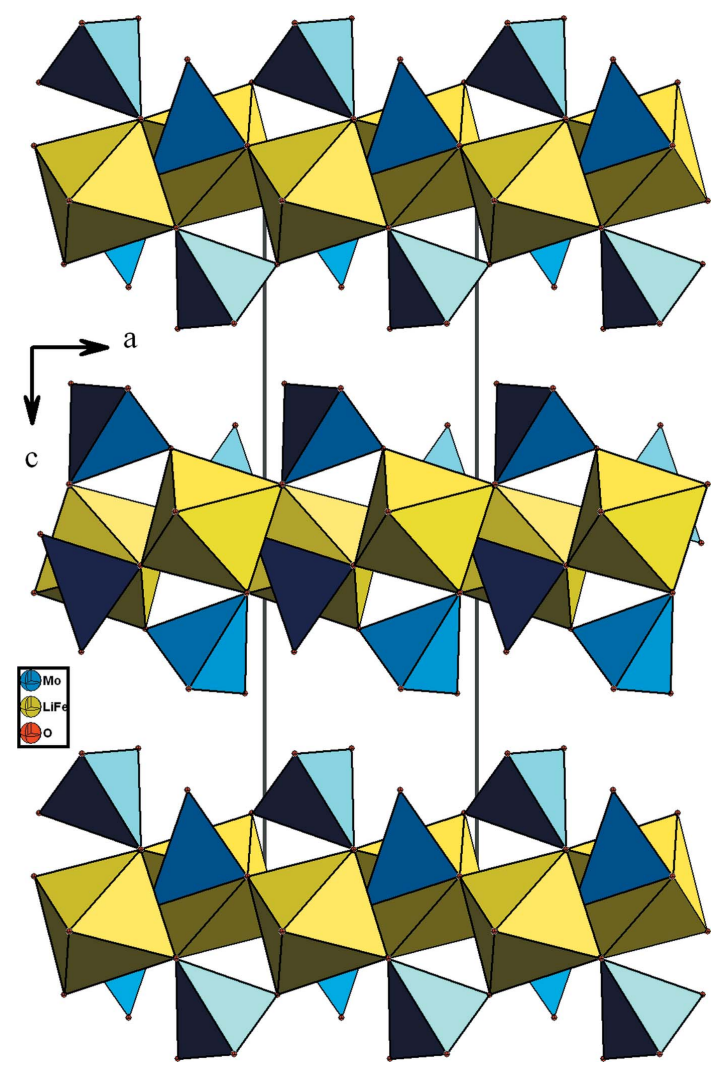

Figure 4

Vue selon $b$, montrant la disposition des couches dans le composé $\left(\mathrm{Na}_{0,4}, \mathrm{Li}_{0,6}\right)\left(\mathrm{Fe}, \mathrm{Li}_{2}\right)\left(\mathrm{MoO}_{4}\right)_{3}$.

le potentiel électrochimique le plus élevé par rapport à l'électrode à hydrogène standard ce qui confère à la batterie une plus haute tension d'où la naissance des batteries rechargeables au lithium $\left(\mathrm{LiFePO}_{4}\right)$ (Padhi et al., 1997) qui sont un dispositif important pour le stockage de l'énergie électrique.

\section{Commentaire structurelle}

L'unité structurale dans la charpente anionique du composé étudié (Fig. 1) est formée de deux octaèdres $M_{6}(M=\mathrm{Fe} / \mathrm{Li})$, disposés en cycle avec deux tétraèdres $\mathrm{MoO}_{4}$ liés par mise en commun de sommets oxygène. Ces unités se regroupent par partage d'arêtes entre octaèdres ainsi que par formation de ponts mixtes de type $M 1-\mathrm{O}-\mathrm{Mo}(M 1=\mathrm{Fe} 1 / \mathrm{Li} 1)$ pour conduire à des rubans disposés selon la direction [100] (Fig. 2). Dans la charpente anionique les octaèdres se lient de deux façons différentes pour conduire à deux types de chaînes. En effet, les octaèdres $M 1 \mathrm{O}_{6}(M 1=\mathrm{Fe} 1 / \mathrm{Li1})$ se regroupent moyennant des faces pour donner naissance à des chaînes de type $\left(M 1 \mathrm{O}_{3}\right)(M 1=\mathrm{Fe} 1 / \mathrm{Li1})$ (Fig. 3a) dans lesquelle la distance métal-métal s'avère très courte de l'ordre $2,57 \AA$ A, qui pourrait prédire certaines propriétés magnétiques au matériau obtenu. Par contre les octaèdres $\mathrm{M}_{2} \mathrm{O}_{6}$ se connectent par mise en commun d'arêtes pour former des chaînes de type $M 2 \mathrm{O}_{4}$ $(M 2=$ Fe2/Li2) (Fig. 3b). Dans ces dernières les distances métal-métal sont situées dans l'interval (3,050-3,197 ̊) 
Tableau 1

Longueurs de liaison sélectionnées.

\begin{tabular}{|c|c|c|c|}
\hline $\mathrm{Na} 1-\mathrm{O} 2$ & 2,181 (4) & $\mathrm{Fe} 2-\mathrm{O} 1^{\mathrm{viii}}$ & $2,126(3)$ \\
\hline $\mathrm{Na} 1-\mathrm{O} 5$ & 2,239 (4) & $\mathrm{Fe} 2-\mathrm{O} 3$ & $2,143(3)$ \\
\hline $\mathrm{Na} 1-\mathrm{O}^{\mathrm{i}}$ & $2,331(5)$ & $\mathrm{Fe} 2-\mathrm{O} 1^{\mathrm{ix}}$ & 2,211 (3) \\
\hline $\mathrm{Fe} 1-\mathrm{O}^{\mathrm{ii}}$ & $2,024(3)$ & Mo1-O1 & $1,764(3)$ \\
\hline $\mathrm{Fe} 1-\mathrm{O} 7^{\mathrm{iii}}$ & $2,033(3)$ & Mo1-O6 & $1,786(4)$ \\
\hline $\mathrm{Fe} 1-\mathrm{O}^{\mathrm{iv}}$ & 2,094 (4) & Mo1-O3 ${ }^{\mathrm{x}}$ & 1,795 (4) \\
\hline $\mathrm{Fe} 1-\mathrm{O}^{\mathrm{v}}$ & 2,107 (4) & Mo2-O5 & $1,735(3)$ \\
\hline $\mathrm{Fe} 2-\mathrm{O} 2$ & 2,042 (3) & $\mathrm{Mo} 2-\mathrm{O}^{\mathrm{xi}}$ & $1,773(3)$ \\
\hline $\mathrm{Fe} 2-\mathrm{O} 4^{\mathrm{vi}}$ & $2,043(3)$ & $\mathrm{Mo} 2-\mathrm{O} 4^{\mathrm{vii}}$ & $1,790(3)$ \\
\hline $\mathrm{Fe} 2-\mathrm{O} 4^{\mathrm{vii}}$ & 2,079 (3) & $\mathrm{Mo} 2-\mathrm{O} 7^{\text {vii }}$ & 1,797 (3) \\
\hline
\end{tabular}

Codes de symétrie: (i) $x-\frac{1}{2}, y,-z+\frac{1}{2}$; (ii) $x-\frac{1}{2},-y+\frac{1}{2},-z+\frac{1}{2}$; (iii) $x-1, y, z$; (iv) $-x+\frac{1}{2},-y+1, z-\frac{1}{2} ; \quad$ (v) $-x+1,-y+1,-z+1$; (vi) $-x+1,-y+1,-z$; (vii) $x, y+1, z$; (viii) $x-1, y, z-1$; (ix) $-x+1,-y+2,-z+1$; (x) $x, y, z+1$; (xi) $x+1, y, z$.

similaires à celles rencontrées dans les matériaux $\mathrm{CsFe}_{5}\left(\mathrm{MoO}_{4}\right)_{7}\left(\mathrm{Namsaraeva}\right.$ et al., 2011) et $\mathrm{K}_{2} \mathrm{Zn}_{2}\left(\mathrm{MoO}_{4}\right)_{3}$ (Gicquel-Mayer \& Perez 1975).

Les chaînes s'associent par partage de sommets avec les tétraèdres $\mathrm{MoO}_{4}$ pour conduire à des couches disposées parallèlement au plan $a b$ (Fig. 4). La jonction de ces couches est assurée, d'une part par insertion des chaînes de type bronze $M 1 \mathrm{O}_{3}(M 1=\mathrm{Fe} 1 / \mathrm{Li} 1)$, et d'autre part par partage de sommet avec les tétraèdres $\mathrm{MoO}_{4}$. Il en résulte une charpente tridimensionnelle possédant des canaux allongés où résident les cations monovalents (Fig. 5).

L'examen des distances $\mathrm{Mo}-\mathrm{O}$ existant dans les tétraèdres $\mathrm{MoO}_{4}$ (tableau 1) sont en bon accord avec celles rencontrées dans la littérature (Klevtsova \& Magarill, 1970; van der Lee et al., 2008).

Par contre, les facteurs métriques des octaèdres $M \mathrm{O}_{6}$ dans la structure s'avèrent des distances moyennes entre celles $\mathrm{Fe}^{3+}-\mathrm{O}$ et $\mathrm{Li}^{+}-\mathrm{O}$ rencontrèes respectivement dans Klevtsova \& Magarill (1970) et van der Lee et al. (2008).

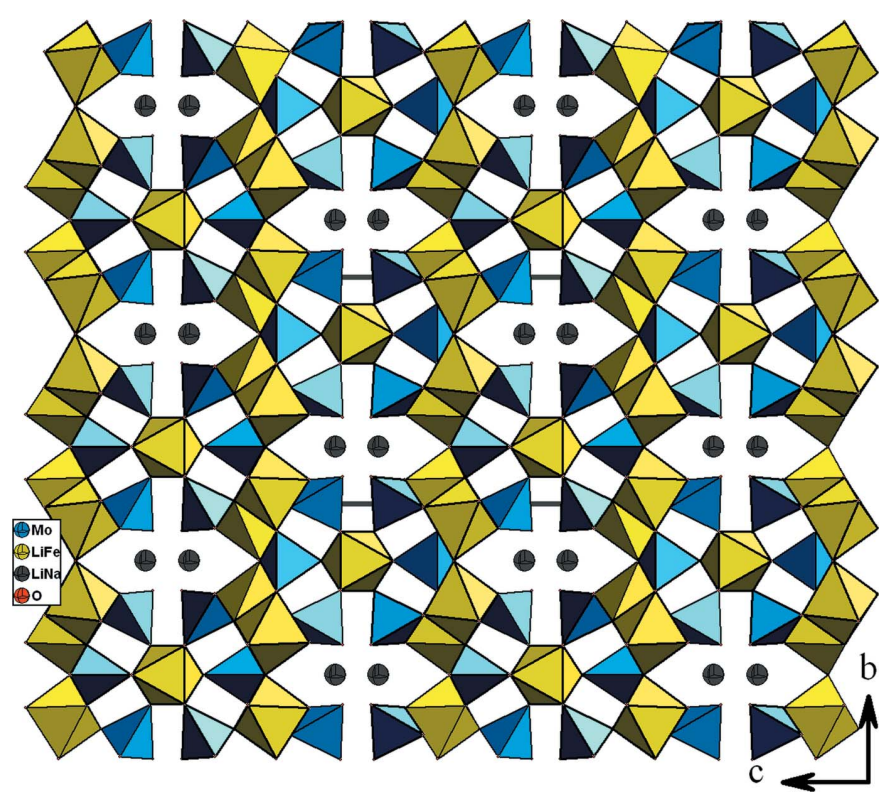

Figure 5

Projection de la structure de $\left(\mathrm{Na}_{0,4}, \mathrm{Li}_{0,6}\right)\left(\mathrm{Fe}, \mathrm{Li}_{2}\right)\left(\mathrm{MoO}_{4}\right)_{3}$, selon $a$, mettant en évidence la disposition des cations alcalins.

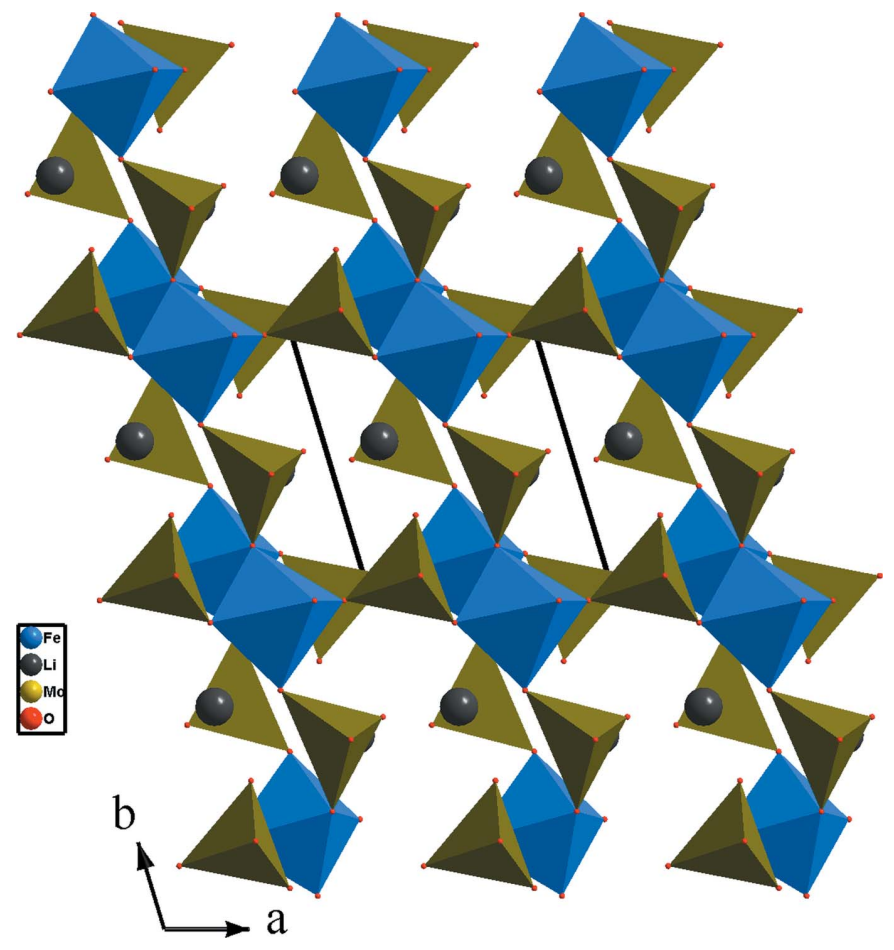

Figure 6

Jonction des polyèdres dans la structure de $\mathrm{LiFe}\left(\mathrm{MoO}_{4}\right)_{2}$.

D'autre part, le calcul des valences de liaisons (BVS), utilisant la formule empirique de Brown (Brown \& Altermatt, 1985; Brown, 2002), conduit aux valeurs des charges des cations suivants: Fe1/Li1 2,73; Fe2/Li2 2,37; Mo1 5,68; Mo2 5,75 et $\mathrm{Na} 1 / \mathrm{Li} 31,06$.

\section{Enquête de base de données}

Dans le cadre d'élaboration de nouveaux molybdates de fer, ainsi que l'amélioration des performances électrochimiques de ces batteries, on a voulu substituer le lithium par le sodium d'où la synthèse de notre nouvelle phase de formulation $\left(\mathrm{Na}_{0,4}, \mathrm{Li}_{0,6}\right)\left(\mathrm{Li}_{2}, \mathrm{Fe}\right)\left(\mathrm{MoO}_{4}\right)_{3}$. Une recherche bibliographique des paramètres de maille dans la base de données Findit (ICSD, 2007) montre que la phase élaborée est isotype à deux structures: $\mathrm{Li}_{3} \mathrm{Fe}\left(\mathrm{MoO}_{4}\right)_{3}$ (Klevtsova \& Magarill, 1970) et $\mathrm{Li}_{3} \mathrm{Ga}\left(\mathrm{MoO}_{4}\right)_{3}$ (van der Lee et al., 2008). Elle est formée d'une charpente tridimensionnelle construite à partir d'octaèdres $M \mathrm{O}_{6}(M=\mathrm{Fe} / \mathrm{Li})$ et des tétraèdres $\mathrm{MoO}_{4}$.

Une comparaison de la structure du composé étudié $\mathrm{Na}_{0,4} \mathrm{Li}_{2,6} \mathrm{Fe}\left(\mathrm{MoO}_{4}\right)_{3}$ avec celle de $\mathrm{LiFeMo}_{2} \mathrm{O}_{8}$ (van der Lee et al., 2008) de système cristallin triclinique (groupe d'espace $P \overline{1}$ ) montre une différence nette dans l'entourage des polyèdres et en particulier les types de chaînes dans la charpente anionique. En effet, on remarque que pour le composé au lithium, chaque molybdate $\mathrm{MoO}_{4}$ permet de relier par partage de sommets deux octaèdres $\mathrm{FeO}_{6}$ différents appartenant à la même chaîne (Fig. 6). Par contre dans le composé étudié chaque molybdate partage trois de ses sommets avec trois octaèdres $\mathrm{M} 2 \mathrm{O}_{6}$ pour relier deux types de chaînes différentes. 


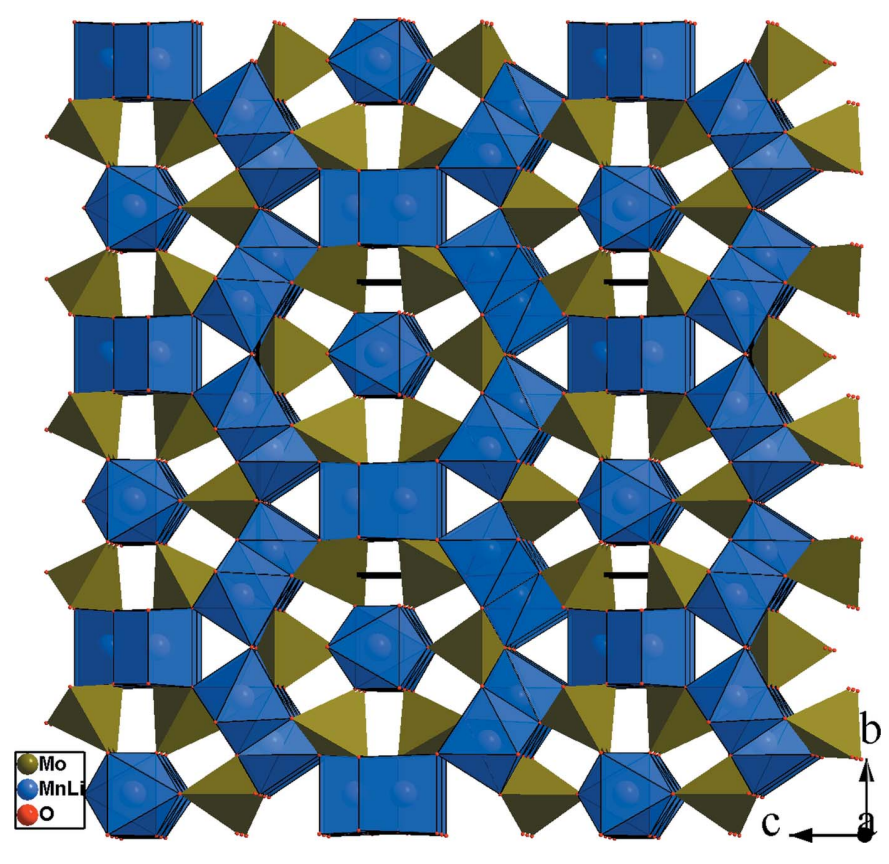

Figure 7

Projection de la structure de $\mathrm{Li}_{1.6} \mathrm{Mn}_{2.2} \mathrm{Mo}_{3} \mathrm{O}_{12}$, selon $a$, montrant la disposition des ions $\mathrm{Mn} / \mathrm{Li}$ dans les octaèdres et dans les bipyramides.

Un autre examen bibliographique nous a conduit vers la série des phases isostructurales suivantes: $\mathrm{Li}_{3} \mathrm{GaMo}_{3} \mathrm{O}_{12}$ (van der Lee et al., 2008), $\mathrm{Li}_{3} \mathrm{FeMo}_{3} \mathrm{O}_{12}$ (Klevtsova \& Magarill, 1970), $\mathrm{Li}_{3} \mathrm{Ti}_{0,75} \mathrm{Mo}_{3} \mathrm{O}_{12}$ (Smit et al., 2008), et $\mathrm{Li}_{1,6} \mathrm{Mn}_{2,2} \mathrm{Mo}_{3} \mathrm{O}_{12}$ (Solodovnikov et al., 1994). Ces dernières cristallisent dans le système orthorhombique (groupe d'espace Pnma) et appartiennent à deux familles différentes, notamment les lyonsites et les bronzes. Elles présentent le même type de charpente anionique, sauf pour la dernière phase $\mathrm{Li}_{1,6} \mathrm{Mn}_{2,2} \mathrm{Mo}_{3} \mathrm{O}_{12}$ on note bien une légère différence dans l'occupation des sites où les ions $(\mathrm{Mn} / \mathrm{Li})$ occupent les sites octaédriques et aussi les cavités bipyramidales (Fig. 7).

\section{Synthèse et cristallisation}

La synthèse de $\mathrm{Na}_{0,4} \mathrm{Li}_{2,6} \mathrm{Fe}\left(\mathrm{MoO}_{4}\right)_{3}$ a été effectuée par réaction à l'état solide, à partir d'un mélange de carbonate de sodium (FLUKA, 71350), carbonate de lithium (AZIENDA CHIMICA, 104094819), de nitrate de fer (Fluka 44949) et de molybdate d'ammonium (Fluka, 69858) pris dans les rapports molaires telques Na:Li:Fe:Mo égaux à 1:2:1:6, respectivement. Après un broyage poussé dans un mortier en agate, le mélange est placé dans un creuset en porcelaine, puis porté dans un premier temps à une température de $673 \mathrm{~K}$ pendant 4 heures, en vue d'éliminer les produits volatils. Un second traitement thermique a été effectué à une température de synthèse proche de la fusion à $1173 \mathrm{~K}$ pendant deux semaines pour favoriser la germination des cristaux. Le résidu final est ensuite ramené à un refroidissement lent de $5 \mathrm{~K} / 12 \mathrm{~h}$ jusqu'à $1123 \mathrm{~K}$ puis rapide $(50 \mathrm{~K} / \mathrm{h})$ jusqu'à la température ambiante. Des cristaux de couleur verdâtre sont séparés à l'eau chaude.
Tableau 2

Détails expérimentaux.

\begin{tabular}{|c|c|}
\hline \multicolumn{2}{|l|}{ Données crystallines } \\
\hline Formule chimique & $\left(\mathrm{Na}_{0,4} \mathrm{Li}_{0,6}\right)\left(\mathrm{FeLi}_{2}\right)\left(\mathrm{MoO}_{4}\right)_{3}$ \\
\hline$M_{\mathrm{r}}$ & 562,91 \\
\hline Système cristallin, groupe d'espace & Orthorhombique, Pnma \\
\hline Température (K) & 298 \\
\hline$a, b, c(\AA)$ & $5,1358(7), 10,5687(9), 17,606(2)$ \\
\hline$V\left(\AA^{3}\right)$ & $955,61(19)$ \\
\hline$Z$ & 4 \\
\hline Type de rayonnement & Мо $K \alpha$ \\
\hline$\mu\left(\mathrm{mm}^{-1}\right)$ & 5,41 \\
\hline Taille des cristaux (mm) & $0,28 \times 0,21 \times 0,14$ \\
\hline \multicolumn{2}{|l|}{ Collection de données } \\
\hline Diffractomètre & Enraf-Nonius CAD-4 \\
\hline Correction d'absorption & $\psi$ scan (North et al., 1968) \\
\hline$T_{\min }, T_{\max }$ & $0,286,0,488$ \\
\hline $\begin{array}{l}\text { Nombre de réflexions mesurées, } \\
\text { indépendantes et observées } \\
{[I>2 \sigma(I)]}\end{array}$ & $2321,1091,1017$ \\
\hline$R_{\text {int }}$ & 0,023 \\
\hline$(\sin \theta / \lambda)_{\max }\left(\AA^{-1}\right)$ & 0,638 \\
\hline \multicolumn{2}{|l|}{ Affinement } \\
\hline$R\left[F^{2}>2 \sigma\left(F^{2}\right)\right], w R\left(F^{2}\right), S$ & $0,023,0,056,1,16$ \\
\hline Nombre de réflexions & 1091 \\
\hline Nombre de paramètres & 101 \\
\hline$\Delta \rho_{\max }, \Delta \rho_{\min }\left(\mathrm{e} \AA^{-3}\right)$ & $0,52,-0,60$ \\
\hline
\end{tabular}

Programmes informatiques: CAD-4 EXPRESS (Duisenberg, 1992; Macíček \& Yordanov, 1992), XCAD4 (Harms \& Wocadlo, 1995), SHELXS97 et SHELXL97 (Sheldrick, 2008), DIAMOND (Brandenburg \& Putz, 1999) et WinGX (Farrugia, 2012).

\section{Affinement}

Détails de donnés crystallines, collection de donnés et affinement sont résumés dans le tableau 2. La structure a été résolu par les méthodes directes (SHELXS97; Sheldrick, 2008), partant de la formule $\mathrm{NaLi}_{2} \mathrm{Fe}\left(\mathrm{MoO}_{4}\right)_{3}$ similaire au composé isotype $\mathrm{Li}_{3} \mathrm{Ga}\left(\mathrm{MoO}_{4}\right)_{3}$. Au départ, l'affinement à été mené avec un taux complet des sites. Un examen de la Fourierdifférence montre des pics d'intensité $(-4,5 \AA$ A $)$ proches des atomes de fer (à $0.47 \AA$ from Fe2). L'utilisation des fonctions SUMP et EADP autorisées par le programme $S H E L X$, pour les couples d'ions $\mathrm{Fe} 1 / \mathrm{Li} 1, \mathrm{Fe} 2 / \mathrm{Li} 2$ et $\mathrm{Na} 1 / \mathrm{Li} 3$ conduit à des ellipsoides bien définis. De plus, les densités d'électrons maximum et minimum restants dans la Fourier-différence sont acceptables et sont situées respectivements à $0.81 \AA$ de $\mathrm{O} 2$ et à $0.43 \AA$ de Li2.

\section{Remerciements}

Les auteurs remercient le Ministère de l'Enseignement Supérieur, de la Recherche Scientifique et de la technologie de la Tunisie pour le financement du laboratoire LMC (code LR01ES11).

\section{Références}

Brandenburg, K. \& Putz, H. (1999). DIAMOND. Crystal Impact GbR, Bonn, Germany.

Brown, I. D. (2002). The Chemical Bond in Inorganic Chemistry The Bond Valence Model. IUCr Monographs on Crystallography, No. 12. Oxford University Press.

Brown, I. D. \& Altermatt, D. (1985). Acta Cryst. B41, 244-247. 
Duisenberg, A. J. M. (1992). J. Appl. Cryst. 25, 92-96.

Farrugia, L. J. (2012). J. Appl. Cryst. 45, 849-854.

Gicquel-Mayer, C. \& Perez, G. (1975). Rev. Chim. Miner. 12, 537-545.

Harms, K. \& Wocadlo, S. (1995). XCAD4. University of Marburg, Allemagne.

ICSD (2007). Version 1.0. ICSD est disponible à FIZ Karlsruhe at http://www.fiz-karlsruhe. de/icsd. html ou http://icsdweb.fiz-karlsruhe. de.

Isupov, V. A. (2005). Ferroelectrics, 322, 83-114.

Jorge, G. A., Capan, C., Ronning, F., Jaime, M., Kenzelmann, M., Gasparovic, G., Broholm, C., Shapiro, A. Ya. \& Demianets, L. A. (2004). Physica B, 354, 297-299.

Klevtsova, R. F. \& Magarill, S. A. (1970). Kristallografiya, 15, 611615.

Klevtsov, P. V. \& Klevtsova, R. F. (1977). Zh. Strukt. Khim. 18, 419439.

Lee, A. van der, Beaurain, M. \& Armand, P. (2008). Acta Cryst. C64, i1-i4.

Macíček, J. \& Yordanov, A. (1992). J. Appl. Cryst. 25, 73-80.
Maczka, M., Pietraszko, A., Saraiva, G. D., Souza Filho, A. G., Paraguassu, W., Lemos, V., Perottoni, C. A., Gallas, M. R., Freire, P. T. C., Tomaszewski, P. E., Melo, F. E. A., Mendes Filho, J. \& Hanuza, J. (2005). J. Phys. Condens. Matter, 17, 6285-6300.

Morozov, V. A., Lazoryak, B. I., Lebedev, O. I., Amelinckx, S. \& Van Tendeloo, G. (2003). J. Solid State Chem. 176, 76-87.

Namsaraeva, T., Bazarov, B., Mikhailova, D., Kuratieva, N., Sarapulova, A., Senyshyn, A. \& Ehrenberg, H. (2011). Eur. J. Inorg. Chem. 18, 2832-2841.

North, A. C. T., Phillips, D. C. \& Mathews, F. S. (1968). Acta Cryst. A24, 351-359.

Padhi, A. K., Nanjundaswamy, K. S. \& Goodenough, J. B. (1997). J. Electrochem. Soc. 144, 1188-1194.

Sheldrick, G. M. (2008). Acta Cryst. A64, 112-122.

Smit, J. P., McDonald, T. M. \& Poeppelmeier, K. R. (2008). Eur. J. Inorg. Chem. 4, 396-400.

Solodovnikov, S. F., Solodovnikova, Z. A., Klevtsova, R. F., Glinskaya, L. A., Klevtsov, P. V. \& Zolotova, E. S. (1994). Zh. Strukt. Khim. 35, 136-144. 


\section{supporting information}

Acta Cryst. (2015). E71, 597-601 [doi:10.1107/S2056989015008737]

\section{Synthèse et étude structrale de lyonsite-type $\left(\mathrm{Na}_{0,4}, \mathrm{Li}_{0,6}\right)\left(\mathrm{Fe}, \mathrm{Li}_{2}\right)\left(\mathrm{MoO}_{4}\right)_{3}$}

\section{Amira Souilem, Mohamed Faouzi Zid et Ahmed Driss}

\section{Computing details}

Data collection: CAD-4 EXPRESS (Duisenberg, 1992; Macíček \& Yordanov, 1992); cell refinement: CAD-4 EXPRESS (Duisenberg, 1992; Macíček \& Yordanov, 1992); data reduction: XCAD4 (Harms \& Wocadlo, 1995); program(s) used to solve structure: SHELXS97 (Sheldrick, 2008); program(s) used to refine structure: SHELXL97 (Sheldrick, 2008); molecular graphics: DIAMOND (Brandenburg \& Putz, 1999); software used to prepare material for publication: WinGX (Farrugia, 2012).

\section{Sodium lithium iron(III) trimolybdate}

Crystal data

$\left(\mathrm{Na}_{0.4} \mathrm{Li}_{0.6}\right)\left(\mathrm{FeLi}_{2}\right)\left(\mathrm{MoO}_{4}\right)_{3}$

$M_{r}=562.91$

Orthorhombic, Pnma

Hall symbol: -P 2 ac $2 \mathrm{n}$

$a=5.1358(7) \AA$

$b=10.5687(9) \AA$

$c=17.606(2) \AA$

$V=955.61(19) \AA^{3}$

$Z=4$

\section{Data collection}

Enraf-Nonius CAD-4 diffractometer

Radiation source: fine-focus sealed tube Graphite monochromator $\omega / 2 \theta$ scans

Absorption correction: $\psi$ scan

(North et al., 1968)

$T_{\min }=0.286, T_{\max }=0.488$

2321 measured reflections

\section{Refinement}

Refinement on $F^{2}$

Least-squares matrix: full

$R\left[F^{2}>2 \sigma\left(F^{2}\right)\right]=0.023$

$w R\left(F^{2}\right)=0.056$

$S=1.16$

1091 reflections

101 parameters

0 restraints

Primary atom site location: structure-invariant direct methods
$F(000)=1041$

$D_{\mathrm{x}}=3.913 \mathrm{Mg} \mathrm{m}^{-3}$

Mo $K \alpha$ radiation, $\lambda=0.71073 \AA$

Cell parameters from 25 reflections

$\theta=11-15^{\circ}$

$\mu=5.41 \mathrm{~mm}^{-1}$

$T=298 \mathrm{~K}$

Prism, green

$0.28 \times 0.21 \times 0.14 \mathrm{~mm}$

1091 independent reflections

1017 reflections with $I>2 \sigma(I)$

$R_{\text {int }}=0.023$

$\theta_{\max }=27.0^{\circ}, \theta_{\min }=2.3^{\circ}$

$h=-6 \rightarrow 6$

$k=-1 \rightarrow 13$

$l=-1 \rightarrow 22$

2 standard reflections every $120 \mathrm{~min}$ intensity decay: $1.3 \%$

Secondary atom site location: difference Fourier map

$w=1 /\left[\sigma^{2}\left(F_{\mathrm{o}}^{2}\right)+(0.0221 P)^{2}+2.5918 P\right]$

where $P=\left(F_{\mathrm{o}}{ }^{2}+2 F_{\mathrm{c}}{ }^{2}\right) / 3$

$(\Delta / \sigma)_{\max }=0.001$

$\Delta \rho_{\max }=0.52 \mathrm{e} \AA^{-3}$

$\Delta \rho_{\min }=-0.60$ e $\AA^{-3}$

Extinction correction: SHELXL, $\mathrm{Fc}^{*}=\mathrm{kFc}\left[1+0.001 \mathrm{xFc}^{2} \lambda^{3} / \sin (2 \theta)\right]^{-1 / 4}$

Extinction coefficient: 0.0030 (2) 


\section{Special details}

Geometry. All e.s.d.'s (except the e.s.d. in the dihedral angle between two 1.s. planes) are estimated using the full covariance matrix. The cell e.s.d.'s are taken into account individually in the estimation of e.s.d.'s in distances, angles and torsion angles; correlations between e.s.d.'s in cell parameters are only used when they are defined by crystal symmetry. An approximate (isotropic) treatment of cell e.s.d.'s is used for estimating e.s.d.'s involving 1.s. planes.

Refinement. Refinement of $F^{2}$ against ALL reflections. The weighted $R$-factor $w R$ and goodness of fit $S$ are based on $F^{2}$, conventional $R$-factors $R$ are based on $F$, with $F$ set to zero for negative $F^{2}$. The threshold expression of $F^{2}>\sigma\left(F^{2}\right)$ is used only for calculating $R$-factors (gt) etc. and is not relevant to the choice of reflections for refinement. $R$-factors based on $F^{2}$ are statistically about twice as large as those based on $F$, and $R$ - factors based on ALL data will be even larger.

Fractional atomic coordinates and isotropic or equivalent isotropic displacement parameters $\left(\hat{A}^{2}\right)$

\begin{tabular}{llllll}
\hline & $x$ & $y$ & $z$ & $U_{\text {iso }} * U_{\text {eq }}$ & Occ. $(<1)$ \\
\hline Mo1 & $0.72100(8)$ & 0.7500 & $0.94298(2)$ & $0.00764(13)$ & \\
Mo2 & $0.78223(6)$ & $0.97805(3)$ & $0.155036(18)$ & $0.00962(13)$ & \\
Fe1 & $0.1092(3)$ & 0.2500 & $0.24993(10)$ & $0.0111(3)$ & 0.40 \\
Li1 & $0.1092(3)$ & 0.2500 & $0.24993(10)$ & $0.0111(3)$ & 0.60 \\
Fe2 & $0.2587(3)$ & $0.92781(16)$ & $0.02588(9)$ & $0.0143(3)$ & 0.30 \\
Li2 & $0.2587(3)$ & $0.92781(16)$ & $0.02588(9)$ & $0.0143(3)$ & 0.70 \\
Na1 & $0.2569(8)$ & 0.7500 & $0.1927(3)$ & $0.0170(9)$ & 0.40 \\
Li3 & $0.2569(8)$ & 0.7500 & $0.1927(3)$ & $0.0170(9)$ & 0.60 \\
O1 & $0.9187(5)$ & $0.8825(3)$ & $0.96258(15)$ & $0.0164(6)$ & \\
O2 & $0.0596(5)$ & $0.8926(3)$ & $0.12403(15)$ & $0.0175(6)$ & \\
O3 & $0.4481(8)$ & 0.7500 & $0.0067(2)$ & $0.0163(8)$ & \\
O4 & $0.5823(6)$ & $0.0138(3)$ & $0.07454(15)$ & $0.0152(6)$ & \\
O5 & $0.5927(6)$ & $0.8810(3)$ & $0.21219(16)$ & $0.0199(6)$ & \\
O6 & $0.6384(8)$ & 0.7500 & $0.8444(2)$ & $0.0142(8)$ & \\
O7 & $0.8577(5)$ & $0.1209(3)$ & $0.20581(15)$ & $0.0142(6)$ & \\
& & & &
\end{tabular}

Atomic displacement parameters $\left(\AA^{2}\right)$

\begin{tabular}{lllllll}
\hline & $U^{11}$ & $U^{22}$ & $U^{33}$ & $U^{12}$ & $U^{13}$ & $U^{23}$ \\
\hline Mo1 & $0.0071(2)$ & $0.0096(2)$ & $0.0062(2)$ & 0.000 & $0.00008(15)$ & 0.000 \\
Mo2 & $0.00910(19)$ & $0.01045(19)$ & $0.00929(18)$ & $-0.00100(12)$ & $-0.00011(11)$ & $-0.00250(12)$ \\
Fe1 & $0.0173(8)$ & $0.0076(7)$ & $0.0084(7)$ & 0.000 & $-0.0018(7)$ & 0.000 \\
Li1 & $0.0173(8)$ & $0.0076(7)$ & $0.0084(7)$ & 0.000 & $-0.0018(7)$ & 0.000 \\
Fe2 & $0.0129(7)$ & $0.0160(8)$ & $0.0140(7)$ & $-0.0010(6)$ & $0.0003(6)$ & $-0.0022(7)$ \\
Li2 & $0.0129(7)$ & $0.0160(8)$ & $0.0140(7)$ & $-0.0010(6)$ & $0.0003(6)$ & $-0.0022(7)$ \\
Na1 & $0.017(2)$ & $0.014(2)$ & $0.020(2)$ & 0.000 & $-0.0016(16)$ & 0.000 \\
Li3 & $0.017(2)$ & $0.014(2)$ & $0.020(2)$ & 0.000 & $-0.0016(16)$ & 0.000 \\
O1 & $0.0166(13)$ & $0.0169(13)$ & $0.0158(13)$ & $-0.0032(12)$ & $-0.0007(11)$ & $0.0005(11)$ \\
O2 & $0.0178(13)$ & $0.0197(15)$ & $0.0150(13)$ & $0.0020(13)$ & $-0.0013(11)$ & $-0.0020(11)$ \\
O3 & $0.0116(18)$ & $0.022(2)$ & $0.0152(18)$ & 0.000 & $0.0010(15)$ & 0.000 \\
O4 & $0.0140(13)$ & $0.0195(14)$ & $0.0122(12)$ & $-0.0029(11)$ & $-0.0001(11)$ & $-0.0004(11)$ \\
O5 & $0.0181(14)$ & $0.0212(15)$ & $0.0204(14)$ & $-0.0054(13)$ & $0.0021(11)$ & $0.0036(12)$ \\
O6 & $0.018(2)$ & $0.0130(19)$ & $0.0115(18)$ & 0.000 & $-0.0017(15)$ & 0.000 \\
O7 & $0.0169(13)$ & $0.0123(13)$ & $0.0134(13)$ & $0.0007(12)$ & $0.0001(10)$ & $-0.0021(11)$ \\
& & & & & &
\end{tabular}


Geometric parameters $(\AA, \stackrel{\circ}{)}$

\begin{tabular}{|c|c|c|c|}
\hline $\mathrm{Na} 1-\mathrm{O} 2^{\mathrm{i}}$ & $2.181(4)$ & $\mathrm{Fe} 2-\mathrm{O} 4^{\mathrm{ix}}$ & $2.043(3)$ \\
\hline $\mathrm{Na} 1-\mathrm{O} 2$ & $2.181(4)$ & $\mathrm{Fe} 2-\mathrm{O} 4^{\mathrm{x}}$ & 2.079 (3) \\
\hline $\mathrm{Na} 1-\mathrm{O} 5$ & $2.239(4)$ & $\mathrm{Fe} 2-\mathrm{O} 1^{\mathrm{xi}}$ & $2.126(3)$ \\
\hline $\mathrm{Na} 1-\mathrm{O} 5^{\mathrm{i}}$ & $2.239(4)$ & $\mathrm{Fe} 2-\mathrm{O} 3$ & $2.143(3)$ \\
\hline $\mathrm{Na} 1-\mathrm{O} 5^{\mathrm{ii}}$ & $2.331(5)$ & $\mathrm{Fe} 2-\mathrm{O} 1^{\mathrm{xii}}$ & $2.211(3)$ \\
\hline $\mathrm{Na} 1-\mathrm{O} 5^{\mathrm{iii}}$ & $2.331(5)$ & Mo1-O1 & $1.764(3)$ \\
\hline $\mathrm{Fe} 1-\mathrm{O} 7^{\mathrm{iv}}$ & $2.024(3)$ & $\mathrm{Mo1}-\mathrm{O} 1^{\mathrm{i}}$ & $1.764(3)$ \\
\hline $\mathrm{Fe} 1-\mathrm{O} 7^{\mathrm{ii}}$ & $2.024(3)$ & Mo1-O6 & $1.786(4)$ \\
\hline $\mathrm{Fe} 1-\mathrm{O}^{\mathrm{v}}$ & $2.033(3)$ & Mo1-O3 ${ }^{\text {xiii }}$ & $1.795(4)$ \\
\hline $\mathrm{Fe} 1-\mathrm{O} 7^{\mathrm{vi}}$ & $2.033(3)$ & $\mathrm{Mo} 2-\mathrm{O} 5$ & $1.735(3)$ \\
\hline $\mathrm{Fe} 1-\mathrm{O}^{\mathrm{vii}}$ & $2.094(4)$ & $\mathrm{Mo} 2-\mathrm{O} 2^{\text {xiv }}$ & $1.773(3)$ \\
\hline $\mathrm{Fe} 1-\mathrm{O6}^{\text {viii }}$ & $2.107(4)$ & $\mathrm{Mo} 2-\mathrm{O} 4^{\mathrm{x}}$ & $1.790(3)$ \\
\hline $\mathrm{Fe} 2-\mathrm{O} 2$ & $2.042(3)$ & $\mathrm{Mo} 2-\mathrm{O} 7^{\mathrm{x}}$ & $1.797(3)$ \\
\hline $\mathrm{O} 1-\mathrm{Mo} 1-\mathrm{O} 1^{\mathrm{i}}$ & $105.12(19)$ & $\mathrm{O} 7^{\mathrm{vi}}-\mathrm{Fe} 1-\mathrm{O}^{\mathrm{vii}}$ & 85.29 (13) \\
\hline $\mathrm{O} 1-\mathrm{Mo1}-\mathrm{O} 6$ & $109.07(11)$ & $\mathrm{O} 7^{\mathrm{iv}}-\mathrm{Fe} 1-\mathrm{O}^{\text {viii }}$ & 85.15 (13) \\
\hline $\mathrm{O} 1$ - & $109.07(11)$ & $\mathrm{O} 7^{\mathrm{ii}}-\mathrm{Fe} 1-\mathrm{O}^{\mathrm{viii}}$ & 85.15 (12) \\
\hline $\mathrm{O} 1-\mathrm{Mo1}-\mathrm{O} 3^{\mathrm{xiii}}$ & $109.09(12)$ & $\mathrm{O} 7^{\mathrm{v}}-\mathrm{Fe} 1-\mathrm{O}^{\mathrm{viii}}$ & 95.14 (12) \\
\hline $\mathrm{O} 1^{\mathrm{i}}-\mathrm{Mo} 1-\mathrm{O} 3^{\mathrm{xiii}}$ & $109.09(12)$ & $\mathrm{O} 7^{\mathrm{vi}}-\mathrm{Fe} 1-\mathrm{O}^{\text {viii }}$ & 95.14 (12) \\
\hline $\mathrm{O} 6-\mathrm{Mo} 1-\mathrm{O}^{\mathrm{xiii}}$ & $114.94(18)$ & $\mathrm{O}^{\mathrm{vii}}-\mathrm{Fe} 1-\mathrm{O}^{\mathrm{viii}}$ & $179.4(2)$ \\
\hline $\mathrm{O} 5-\mathrm{Mo} 2-\mathrm{O} 2^{\mathrm{xiv}}$ & $109.15(14)$ & $\mathrm{O} 2-\mathrm{Fe} 2-\mathrm{O} 4^{\mathrm{ix}}$ & $170.95(15)$ \\
\hline $\mathrm{O} 5-\mathrm{Mo} 2-\mathrm{O} 4^{\mathrm{x}}$ & $105.19(13)$ & $\mathrm{O} 2-\mathrm{Fe} 2-\mathrm{O} 4^{\mathrm{x}}$ & $97.55(13)$ \\
\hline $\mathrm{O} 2^{\mathrm{xiv}}-\mathrm{Mo} 2-\mathrm{O} 4^{\mathrm{x}}$ & $108.95(13)$ & $\mathrm{O} 4^{\mathrm{ix}}-\mathrm{Fe} 2-\mathrm{O} 4^{\mathrm{x}}$ & 84.55 (13) \\
\hline $\mathrm{O} 5-\mathrm{Mo} 2-\mathrm{O}^{\mathrm{x}}$ & $109.21(13)$ & $\mathrm{O} 2-\mathrm{Fe} 2-\mathrm{O}^{\mathrm{xi}}$ & $89.50(12)$ \\
\hline $\mathrm{O} 2^{\mathrm{xiv}}-\mathrm{Mo} 2-\mathrm{O} 7^{\mathrm{x}}$ & $114.08(13)$ & $\mathrm{O} 4^{\mathrm{ix}}-\mathrm{Fe} 2-\mathrm{O} 1^{\mathrm{xi}}$ & $86.71(12)$ \\
\hline $\mathrm{O} 4^{\mathrm{x}}-\mathrm{Mo} 2-\mathrm{O}^{\mathrm{x}}$ & $109.88(12)$ & $\mathrm{O} 4^{\mathrm{x}}-\mathrm{Fe} 2-\mathrm{O} 1^{\mathrm{xi}}$ & $166.17(14)$ \\
\hline $\mathrm{O} 7^{\mathrm{iv}}-\mathrm{Fe} 1-\mathrm{O} 7^{\mathrm{ii}}$ & $84.76(17)$ & $\mathrm{O} 2-\mathrm{Fe} 2-\mathrm{O} 3$ & $101.57(14)$ \\
\hline $\mathrm{O} 7^{\mathrm{iv}}-\mathrm{Fe} 1-\mathrm{O}^{\mathrm{v}}$ & $179.64(16)$ & $\mathrm{O} 4^{\mathrm{ix}}-\mathrm{Fe} 2-\mathrm{O} 3$ & 86.97 (14) \\
\hline $\mathrm{O} 7^{\mathrm{ii}-}-\mathrm{Fe} 1-\mathrm{O}^{\mathrm{v}}$ & $95.46(12)$ & $\mathrm{O} 4^{x}-\mathrm{Fe} 2-\mathrm{O} 3$ & $94.90(14)$ \\
\hline $\mathrm{O} 7^{\mathrm{iv}}-\mathrm{Fe} 1-\mathrm{O} 7^{\mathrm{vi}}$ & $95.46(12)$ & $\mathrm{O} 1^{\mathrm{xi}}-\mathrm{Fe} 2-\mathrm{O} 3$ & 95.33 (14) \\
\hline 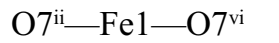 & $179.64(16)$ & $\mathrm{O} 2-\mathrm{Fe} 2-\mathrm{O} 1^{\mathrm{xii}}$ & $83.18(12)$ \\
\hline $\mathrm{O} 7^{\mathrm{v}}-\mathrm{Fe} 1-\mathrm{O} 7^{\mathrm{vi}}$ & $84.32(17)$ & $\mathrm{O} 4^{\mathrm{ix}}-\mathrm{Fe} 2-\mathrm{O} 1^{\mathrm{xii}}$ & $88.31(13)$ \\
\hline $\mathrm{O}^{\mathrm{iv}}-\mathrm{Fe} 1-\mathrm{O}^{\mathrm{vii}}$ & $94.41(13)$ & $\mathrm{O} 4^{x}-\mathrm{Fe} 2-\mathrm{O} 1^{\mathrm{xii}}$ & $83.98(12)$ \\
\hline $\mathrm{O} 7^{\mathrm{ii}}-\mathrm{Fe} 1-\mathrm{O}^{\mathrm{vii}}$ & $94.41(13)$ & 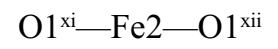 & $85.06(12)$ \\
\hline $\mathrm{O} 7^{\mathrm{v}}-\mathrm{Fe} 1-\mathrm{O}^{\mathrm{vii}}$ & $85.29(13)$ & $\mathrm{O} 3-\mathrm{Fe} 2-\mathrm{O} 1^{\mathrm{xii}}$ & $175.23(15)$ \\
\hline
\end{tabular}

Symmetry codes: (i) $x,-y+3 / 2, z$; (ii) $x-1 / 2, y,-z+1 / 2$; (iii) $x-1 / 2,-y+3 / 2,-z+1 / 2$; (iv) $x-1 / 2,-y+1 / 2,-z+1 / 2$; (v) $x-1, y, z$; (vi) $x-1,-y+1 / 2, z$; (vii) $-x+1 / 2,-y+1, z-1 / 2$; (viii) $-x+1,-y+1,-z+1$; (ix) $-x+1,-y+1,-z$; (x) $x, y+1, z$; (xi) $x-1, y, z-1$; (xii) $-x+1,-y+2,-z+1$; (xiii) $x, y, z+1$; (xiv) $x+1, y, z$. 